\title{
STABILITY AND APPROXIMATIONS OF AN ACOUSTIC-STRUCTURE MODEL
}

\author{
Fariba Fahroo \\ Naval Postgraduate School \\ Mathematics Department, Code MA/Ff \\ Monterey, CA 93943-5216, USA* \\ fahroo@math.nps.navy.mil \\ Chunming Wang \\ University of Southern California \\ Department of Mathematics \\ Los Angeles, CA 90089-1113, USA ${ }^{\dagger}$ \\ cwang@optimize2.usc.edu
}

\begin{abstract}
We consider uniform exponential stability and preservation of this property under approximations for a coupled acoustic- structure system with "porous" damped boundary conditions. This system consists of a 2-D acoustic cavity whose portion of its boundary is modeled as a flexible beam. Using a multiplier technique we show that the "porous" boundary condition provides enough damping to yield uniform exponential stability of the model. We also present a polynomial-based Galerkin numerical scheme which results in approximate solutions with a uniform rate of decay.
\end{abstract}

Key Words: acoustic-structure models, multiplier technique, exponential stability, Galerkin method.

AMS subject classification: 35L05, 93D20, 65N12, 65N30.

\section{INTRODUCTION}

In this paper, we consider a two dimensional acoustic-structure system which consists of a rectangular acoustic cavity whose two sides are bounded by EulerBernoulli beams. The acoustic cavity is $\Omega=(-1,1) \times(-1,1)$ and the two beams are located at the sides $x=-1$ and $y=-1$.

\footnotetext{
*The research of this author was supported in part by the Naval Postgraduate School under a faculty development grant.

${ }^{\dagger}$ The research of this author was supported in part by the Air Force Office of Scientific Research under grant AFOSR-90-0091
}

The original version of this chapter was revised: The copyright line was incorrect. This has been corrected. The Erratum to this chapter is available at DOI: 10.1007/978-0-387-35359-3_40 
The model systems are given by the following partial differential equations (see [4]): for $t>0$

$$
\begin{aligned}
\partial_{t}^{2} \phi(t, x, y) & =\partial_{x}^{2} \phi(t, x, y)+\partial_{y}^{2} \phi(t, x, y), \quad(x, y) \in \Omega, \\
\partial_{t}^{2} u_{1}(t, x) & =-\partial_{x}^{4}\left(E u_{1}(t, x)+C \partial_{t} u_{1}(t, x)\right)+\partial_{t} \phi(t, x,-1), \quad x \in(-1,1), \\
\partial_{t}^{2} u_{2}(t, y) & =-\partial_{y}^{4}\left(E u_{2}(t, y)+C \partial_{t} u_{2}(t, y)\right)+\partial_{t} \phi(t,-1, y), \quad y \in(-1,1),
\end{aligned}
$$

where $\phi$ is the velocity potential function for the acoustic cavity and $u_{1}, u_{2}$ are the transversal displacements of the beams numbered 1 and 2 , respectively. The velocity transmission at the interface between the acoustic cavity and the beams is modeled by

$$
\begin{array}{ll}
\partial_{y} \phi(t, x,-1)=\partial_{t} u_{1}(t, x)+\alpha_{1} \partial_{t} \phi(t, x,-1), & x \in(-1,1) \\
\partial_{x} \phi(t,-1, y)=\partial_{t} u_{2}(t, y)+\alpha_{2} \partial_{t} \phi(t,-1, y), & y \in(-1,1) .
\end{array}
$$

The coefficients $\alpha_{i}, i=1,2$ represent the velocity loss factor. We note that if $\alpha_{i}=0$, the boundary conditions above correspond to the continuity of the velocity at the boundaries. Additional boundary conditions are given by

$$
\begin{array}{ll}
\phi(t, x, 1)=\phi(t, 1, y)=0, & x, y \in(-1,1), \\
u_{1}(t, x)=\partial_{x} u_{1}(t, x)=0, & x=-1,1, \\
u_{2}(t, y)=\partial_{y} u_{2}(t, y)=0, & y=-1,1 .
\end{array}
$$

In this work we present a summary of the results obtained in [4] on wellposedness and uniform exponential stability of the solutions to the equations (1.1-1.8). We also present a polynomial-based Galerkin scheme that preserves the exponential stability of the solutions under approximations. The model presented here is a modification of the one presented first in [3]. The main difference lies in substituting the condition of continuity of normal velocities of the structure and the acoustic field at the boundary of the acoustic cavity used in [3] by boundary conditions (1.4)-(1.5) that allow for a difference in the two velocities proportional to the acoustic pressure. Recently, several studies have appeared in literature that analyze the stability properties of different models derived from the one in [3], (see $[1,2,6,7]$ ). In the same vein the work here also studies the stability of a new model for acoustic-structure interactions, but in addition provides a framework that can be used for proving the existence of a uniform rate of decay for the approximate solutions obtained from a Galerkin method. Since the model equations presented here arise in active noise control problems, for implementation of the linear quadratic regulator control algorithm numerical approximation of the solutions and their exponential stability is of utmost importance. In this regard, this framework facilitates the study of convergence of the approximate Riccati operators and the feedback controls to the infinite-dimensional ones.

\section{WELL-POSEDNESS OF THE MODEL}

For these model equations the state space $\mathcal{H}$ is

$$
\mathcal{H}=V_{a} \times H_{a} \times V_{b} \times H_{b} \times V_{b} \times H_{b}
$$


where the Hilbert spaces $H_{a}=L^{2}((-1,1) \times(-1,1)), H_{b}=L^{2}(-1,1)$ and the Hilbert spaces $V_{a}$ and $V_{b}$ are defined by

$$
\begin{aligned}
& V_{a}=\left\{\phi \in H^{1}((-1,1) \times(-1,1)): \phi(x, 1)=\phi(1, y)=0, \forall x, y \in(-1,1)\right\}, \\
& V_{b}=\left\{u \in H^{2}(-1,1): u(s)=u^{\prime}(s)=0, \text { for } s=-1,1\right\},
\end{aligned}
$$

with inner products defined by

$$
\langle\phi, \tilde{\phi}\rangle_{V_{a}}=\int_{\Omega} \nabla \phi(x, y) \cdot \nabla \tilde{\phi}(x, y) d x d y, \quad\langle u, \tilde{u}\rangle_{V_{b}}=\int_{-1}^{1} E u^{\prime \prime}(s) \tilde{u}^{\prime \prime}(s) d s .
$$

A solution of the variational form of the model equations is a $\mathcal{H}$ valued function of time $t, \xi(t)=\left(\phi(t), \theta(t), u_{1}(t), v_{1}(t), u_{2}(t), v_{2}(t)\right)$, which satisfies the equation

$$
\frac{d}{d t}\langle\xi(t), \tilde{\xi}\rangle_{\mathcal{H}}=\sigma(\xi(t), \tilde{\xi})
$$

for all $\tilde{\xi}=\left(\tilde{\phi}, \tilde{\theta}, \tilde{u}_{1}, \tilde{v}_{1}, \tilde{u}_{2}, \tilde{v}_{2}\right) \in \mathcal{V}$ and where the Hilbert space $\mathcal{V}$ is defined as $\mathcal{V}=V_{a} \times V_{a} \times V_{b} \times V_{b} \times V_{b} \times V_{b}$ and the sesquilinear form $\sigma$ on $\mathcal{V} \times \mathcal{V}$ is defined by

$$
\begin{aligned}
\sigma(\xi, t x i)=\sigma_{a}(\theta, \tilde{\phi})-\sigma_{a}(\phi, \tilde{\theta})-\rho_{1}\left(v_{1}, \tilde{\theta}\right)-\rho_{2}\left(v_{2}, \tilde{\theta}\right)-\gamma_{a}(\theta, \tilde{\theta}) \\
+\sigma_{b}\left(v_{1}, \tilde{u}_{1}\right)-\sigma_{b}\left(u_{1}, \tilde{v}_{1}\right)+\rho_{1}\left(\tilde{v}_{1}, \theta\right)-\gamma_{b}\left(v_{1}, \tilde{v}_{1}\right) \\
+\sigma_{b}\left(v_{2}, \tilde{u}_{2}\right)-\sigma_{b}\left(u_{2}, \tilde{v}_{2}\right)+\rho_{2}\left(\tilde{v}_{2}, \theta\right)-\gamma_{b}\left(v_{2}, \tilde{v}_{2}\right) .
\end{aligned}
$$

The above sesquilinear forms are defined by

$$
\begin{aligned}
& \sigma_{a}(\hat{\phi}, \tilde{\phi})=\int_{\Omega} \nabla \hat{\phi}(x, y) \cdot \nabla \tilde{\phi}(x, y) d x d y \\
& \gamma_{a}(\hat{\phi}, \tilde{\phi})=\int_{-1}^{1} \alpha_{1} \hat{\phi}(x,-1) \tilde{\phi}(x,-1) d x+\int_{-1}^{1} \alpha_{2} \hat{\phi}(-1, y) \tilde{\phi}(-1, y) d y, \\
& \sigma_{b}(\hat{u}, \tilde{u})=\int_{-1}^{1} E \hat{u}^{\prime \prime}(s) \tilde{u}^{\prime \prime}(s) d s, \quad \gamma_{b}(\hat{u}, \tilde{u})=\int_{-1}^{1} C \hat{u}^{\prime \prime}(s) \tilde{u}^{\prime \prime}(s) d s \\
& \rho_{1}(\tilde{u}, \tilde{\phi})=\int_{-1}^{1} \tilde{u}(x) \tilde{\phi}(x,-1) d x, \quad \rho_{2}(\tilde{u}, \tilde{\phi})=\int_{-1}^{1} \tilde{u}(y) \tilde{\phi}(-1, y) d y .
\end{aligned}
$$

We define an unbounded linear operator $\mathcal{A}: \mathcal{D}(\mathcal{A}) \subset \mathcal{H} \mapsto \mathcal{H}$ as follows:

$\mathcal{D}(\mathcal{A})=\left\{\begin{array}{l}\xi=\left(\phi, \theta, u_{1}, v_{1}, u_{2}, v_{2}\right) \in \mathcal{V}: \exists \hat{\xi}=\left(\hat{\phi}, \hat{\theta}, \hat{u}_{1}, \hat{v}_{1}, \hat{u}_{2}, \hat{v}_{2}\right) \in \mathcal{H}, \quad \text { such that } \\ \sigma(\xi, \tilde{\xi})=\langle\hat{\xi}, \tilde{\xi}\rangle_{\mathcal{H}} \text { for all } \tilde{\xi}=\left(\tilde{\phi}, \tilde{\theta}, \tilde{u}_{1}, \tilde{v}_{1}, \tilde{u}_{2}, \tilde{v}_{2}\right) \in \mathcal{V} .\end{array}\right\}$,

and for all $\xi \in \mathcal{D}(\mathcal{A}), \mathcal{A} \xi=\hat{\xi}$ where $\hat{\xi}=\left(\hat{\phi}, \hat{\theta}, \hat{u}_{1}, \hat{v}_{1}, \hat{u}_{2}, \hat{v}_{2}\right)$ satisfies $\sigma(\xi, \tilde{\xi})=$ $\langle\hat{\xi}, \tilde{\xi}\rangle_{\mathcal{H}}$, for all $\tilde{\xi}=\left(\tilde{\phi}, \tilde{\theta}, \tilde{u}_{1}, \tilde{v}_{1}, \tilde{u}_{2}, \tilde{v}_{2}\right) \in \mathcal{V}$.

The main result of this section is the following theorem.

Theorem 2.1 The operator $\mathcal{A}$ is the infinitesimal generator of a $C_{0}$-semigroup of contractions denoted by $e^{\mathcal{A} t}$ in $\mathcal{H}$ and for any $\xi_{0}=\left(\phi_{0}, \theta_{0}, u_{1,0}, v_{1,0}, u_{2,0}, v_{2,0}\right) \in$ $\mathcal{D}(\mathcal{A})$, the function $\xi(t)=e^{\mathcal{A} t} \xi_{0}$ is the unique weak solution of (1.10) with initial value $\xi_{0}$.

The proof of the above theorem is based on the Lumer-Phillips theorem. The conditions in the Lumer-Phillips theorem are established through the following lemmas, (see [4]). 
Lemma 2.1 The operator $\mathcal{A}$ is dissipative in $\mathcal{H}$.

Lemma 2.2 For all real number $\lambda>0$, the operator $\mathcal{A}-\lambda I: \mathcal{D}(\mathcal{A}) \subset \mathcal{H} \mapsto \mathcal{H}$ is onto.

\section{UNIFORM EXPONENTIAL STABILITY OF THE MODEL}

In this section we outline the proof for existence of constants $M \geq 0$ and $\omega>0$ such that

$$
\left\|e^{\mathcal{A} t} \xi_{0}\right\|_{\mathcal{H}} \leq M e^{-\omega t}\left\|\left(\xi_{0}\right)\right\|_{\mathcal{H}},
$$

for all $\xi_{0}=\left(\phi_{0}, \theta_{0}, u_{1,0}, v_{1,0}, u_{2,0}, v_{2,0}\right) \in \mathcal{H}$. Our approach is based on the use of a multiplier functional $Q(t)$ defined by

$$
\begin{aligned}
Q(t)= & \frac{t}{2}\|\xi(t)\|_{\mathcal{H}}^{2}+\int_{-1}^{1} u_{1}(t, x) v_{1}(t, x) d x+\int_{-1}^{1} u_{2}(t, y) v_{2}(t, y) d y \\
& +\int_{\Omega}\left[2(x-1) \partial_{x} \phi(t, x, y)+2(y-1) \partial_{y} \phi(t, x, y)+\phi(t, x, y)\right] \theta(t, x, y) d x d y
\end{aligned}
$$

It is easy to see that there exists a constant $T$ such that for all $t \geq T$,

$$
\frac{t}{4} E(t) \leq Q(t) \leq t E(t)
$$

where $E(t)=\|\xi(t)\|_{\mathcal{H}}^{2}$. The proof of the inequality (1.12) depends on showing that there exists a constant $T^{\prime}$ such that for all $t \geq T^{\prime}, Q(t) \leq Q\left(T^{\prime}\right)$. Therefore, for all $t \geq \max \left(T, T^{\prime}\right)$, we have $\frac{t}{4} E(t) \leq Q\left(T^{\prime}\right) \leq T^{\prime} E\left(T^{\prime}\right)$. As a result, we have $E(t) \leq \frac{4}{t} T^{\prime}\left\|e^{\mathcal{A} T^{\prime}}\right\|_{L(\mathcal{H})}^{2} \cdot\left\|\xi_{0}\right\|_{\mathcal{H}}^{2}$. Using the semigroup property of $e^{\mathcal{A} t}$, we obtain the exponential decay given in inequality (1.12). In order to show that $Q$ is bounded, we shall show $Q^{\prime}(t)$ is negative for all $t>T^{\prime}$ for some constant $T^{\prime}$. First, take $\xi_{0} \in \mathcal{D}(\mathcal{A})$ and let $\xi(t)=e^{\mathcal{A} t} \xi_{0}$.

We consider the function $Q(t)$. Using the differentiability of the function $\left(\phi(\cdot), \theta(\cdot), u_{1}(\cdot), v_{1}(\cdot), u_{2}(\cdot), v_{2}(\cdot)\right)$, we obtain

$$
\begin{aligned}
& \frac{d}{d t} Q((t)=\frac{1}{2}\|\xi(t)\|_{\mathcal{H}}^{2}+t\langle\mathcal{A} \xi(t), \xi(t)\rangle_{\mathcal{H}} \\
& \quad+\int_{-1}^{1} \partial_{t} u_{1}(t, x) v_{1}(t, x) d x+\int_{-1}^{1} u_{1}(t, x) \partial_{t} v_{1}(t, x) d x \\
& \quad+\int_{-1}^{1} \partial_{t} u_{2}(t, y) v_{2}(t, y) d y+\int_{-1}^{1} u_{2}(t, y) \partial_{t} v_{2}(t, y) d y \\
& \quad+\int_{\Omega} \partial_{t} \phi(t, x, y) \theta(t, x, y) d x d y+\int_{\Omega} \phi(t, x, y) \partial_{t} \theta(t, x, y) d x d y \\
& \quad+\int_{\Omega}\left[2(x-1) \partial_{x} \phi(t, x, y)+2(y-1) \partial_{y} \phi(t, x, y)\right] \partial_{t} \theta(t, x, y) d x d y \\
& \quad+\int_{\Omega} \partial_{t}\left[2(x-1) \partial_{x} \phi(t, x, y)+2(y-1) \partial_{y} \phi(t, x, y)\right] \theta(t, x, y) d x d y
\end{aligned}
$$


From the definition of the operator $\mathcal{A}$, integration by parts and by rearranging the terms, we obtain

$$
\begin{aligned}
\frac{d}{d t} Q(t)= & \frac{1}{2} \int_{\Omega} \nabla \phi(t, x, y) \cdot \nabla \phi(t, x, y) d x d y+\int_{\Omega} \phi(t, x, y) \Delta \phi(t, x, y) d x d y \\
& +\int_{\Omega}\left[2(x-1) \partial_{x} \phi(t, x, y)+2(y-1) \partial_{y} \phi(t, x, y)\right] \Delta \phi(t, x, y) d x d y \\
& +\int_{\Omega}\left[2(x-1) \partial_{x} \theta(t, x, y)+2(y-1) \partial_{y} \theta(t, x, y)\right] \theta(t, x, y) d x d y \\
& +\frac{3}{2} \int_{\Omega} \theta^{2}(t, x, y) d x d y-t \int_{-1}^{1} \alpha \theta^{2}(t, x,-1) d x-t \int_{-1}^{1} \alpha \theta^{2}(t,-1, y) d y \\
& +\frac{3}{2} \int_{-1}^{1} v_{1}^{2}(t, x) d x+\frac{3}{2} \int_{-1}^{1} v_{2}^{2}(t, y) d y \\
& -\frac{1}{2} \int_{-1}^{1} E\left(\partial_{x}^{2} u_{1}(t, x)\right)^{2} d x-\frac{1}{2} \int_{-1}^{1} E\left(\partial_{y}^{2} u_{2}(t, y)\right)^{2} d y \\
& +(-t C) \int_{-1}^{1}\left(\partial_{x}^{2} v_{1}(t, x)\right)^{2} d x+(-t C) \int_{-1}^{1}\left(\partial_{y}^{2} v_{2}(t, y)\right)^{2} d y \\
& -C \int_{-1}^{1} \partial_{x}^{2} v_{1}(t, x) \partial_{x}^{2} u_{1}(t, x) d x-C \int_{-1}^{1} \partial_{y}^{2} v_{2}(t, y) \partial_{y}^{2} u_{2}(t, y) d y \\
& +\int_{-1}^{1} \theta(t, x,-1) u_{1}(t, x) d x+\int_{-1}^{1} \theta(t,-1, y) u_{2}(t, y) d y=\sum_{k=1}^{17} I_{k},
\end{aligned}
$$

where $I_{k}$ is the $k$-th integral in the above equality. It is easy to see that there exists $T_{1}>0$ such that for all $t>T_{1}$,

$$
\begin{aligned}
\sum_{k=8}^{15} I_{k} \leq & -\frac{1}{4} \int_{-1}^{1} E\left(\partial_{x}^{2} u_{1}(t, x)\right)^{2} d x-\frac{1}{4} \int_{-1}^{1} E\left(\partial_{y}^{2} u_{2}(t, y)\right)^{2} d y \\
& -\frac{t}{2} \int_{-1}^{1}\left(\partial_{x}^{2} v_{1}(t, x)\right)^{2} d x-\frac{t}{2} \int_{-1}^{1}\left(\partial_{y}^{2} v_{2}(t, y)\right)^{2} d y
\end{aligned}
$$

Using the divergence theorem, we can also show that there exists a constant $T_{2}$ such that for all $t>T_{2}$

$$
I_{4}+I_{5} \leq \frac{t \alpha}{2} \int_{-1}^{1} \theta^{2}(t, x,-1) d x+\frac{t \alpha}{2} \int_{-1}^{1} \theta^{2}(t,-1, y) d y .
$$

On the other hand, for the terms $I_{16}$ and $I_{17}$, it is easy to see that

$$
\begin{aligned}
& \int_{-1}^{1} \theta(t, x,-1) u_{1}(t, x) d x+\int_{-1}^{1} \theta(t,-1, y) u_{2}(t, y) d y \\
\leq \quad & \int_{-1}^{1} u_{1}^{2}(t, x) d x+\epsilon \int_{-1}^{1} u_{2}^{2}(t, y) d x \\
& +\frac{1}{\epsilon} \int_{-1}^{1} \theta^{2}(t, x,-1) d x+\frac{1}{\epsilon} \int_{-1}^{1} \theta^{2}(t,-1, y) d y .
\end{aligned}
$$


For the first 3 terms, we use a sequence of functions $\phi_{n} \in H^{2}(\Omega) \bigcap V_{a}$ that approximate $\phi$ and also have enough smoothness to justify application of the divergence theorem, (see [4]). Therefore, one can show there exists a constant $T_{3}$ such that for all $t>T_{3}$,

$$
\begin{aligned}
I_{1}+I_{2}+I_{3} \leq & \frac{t \alpha}{2} \int_{-1}^{1} \theta^{2}(t, x,-1) d x+\frac{t \alpha}{2} \int_{-1}^{1} \theta^{2}(t,-1, y) d y \\
& +\frac{t}{2} \int_{-1}^{1}\left(\partial_{x}^{2} v_{1}(t, x)\right)^{2} d x+\frac{t}{2} \int_{-1}^{1}\left(\partial_{y}^{2} v_{2}(t, y)\right)^{2} d y .
\end{aligned}
$$

By combining all these results, we get the desired uniform exponential stability.

\section{GALERKIN APPROXIMATIONS}

The Galerkin approximation of the variational form of the model equations requires the use of polynomial functions for the approximation of $V_{a}$. In particular, let $H_{a}^{N}$ be a subspace of $V_{a}$ given by

$$
H_{a}^{N}=\left\{\begin{array}{l}
\phi^{N}: \phi^{N}(x, y) \in \operatorname{span}\left\{x^{k} y^{j}\right\}_{0 \leq k \leq N_{x}, 0 \leq j \leq N_{y}, \quad \text { and }} \\
\phi^{N}(x, 1)=\phi^{N}(1, y)=0, \text { for all } x, y \in(-1,1)
\end{array}\right\}
$$

where $N_{x}$ and $N_{y}$ are positive integers. There are no special restriction on the selection of the subspaces $H_{b}^{N}$ of $V_{b}$ for the discussion of the exponential stability of solutions of the finite dimensional approximations of the model equations. A solution $\xi^{N}(t)=\left(\phi^{N}(t), \theta^{N}(t), u_{1}^{N}(t), v_{1}^{N}(t), u_{2}^{N}(t), v_{2}^{N}(t)\right)$ to the Galerkin approximation on the subspace $\mathcal{H}^{N}$ defined by

$$
\mathcal{H}^{N}=H_{a}^{N} \times H_{a}^{N} \times H_{b}^{N} \times H_{b}^{N} \times H_{b}^{N} \times H_{b}^{N}
$$

is a $\mathcal{H}^{N}$ valued function of time $t$ which satisfies the equation:

$$
\frac{d}{d t}\left\langle\xi^{N}(t), \tilde{\xi}^{N}\right\rangle_{\mathcal{H}}=\sigma\left(\xi^{N}(t), \tilde{\xi}^{N}\right)
$$

for all $\tilde{\xi}^{N}=\left(\tilde{\phi}^{N}, \tilde{\theta}^{N}, \tilde{u}_{1}^{N}, \tilde{v}_{1}^{N}, \tilde{u}_{2}^{N}, \tilde{v}_{2}^{N}\right) \in \mathcal{H}^{N}$. The equation above is a linear evolution equation in $\mathcal{H}^{N}$. As a consequence, there exists a semi-group $S^{N}(t)$ of linear operators on $\mathcal{H}^{N}$ such that for every given initial state vector $\xi_{0}^{N}=$ $\left(\phi_{0}^{N}, \theta_{0}^{N}, u_{1,0}^{N}, v_{1,0}^{N}, u_{2,0}^{N}, v_{2,0}^{N}\right) \in \mathcal{H}^{N}$ the function defined by $\xi^{N}(t)=S^{N}(t) \xi_{0}^{N}$ is a solution of the dynamical equation in $\mathcal{H}^{N}$. The main result on the uniform exponential decay rate of the approximate solution semigroup $S^{N}(t)$ is given by the following theorem.

Theorem 4.1 For the polynomial based Galerkin scheme there exist constants $L \geq 1$ and $\beta>0$ such that

$$
\left\|S^{N}(t)\right\|_{L(\mathcal{H} N} \leq L L e^{-\beta t}
$$

for all $t \geq 0$ and for all $N$.

For the proof of this theorem, see [5]. 
For computational purposes, we represent the model equations in $\mathcal{H}^{N}$ in their coordinate vector form with respect to selected bases for $H_{a}^{N}$ and $H_{b}^{N}$ which are subspaces of polynomials. For this purpose, we let $\left\{w_{i}^{n}\right\}_{i=1}^{n}$ be a basis for $H_{b}^{N}$ and let $\left\{\psi_{k}^{m}\right\}_{k=1}^{m}$ denote the 2-D polynomial basis functions which span $H_{a}^{N}$. By expanding the components of the approximate state vector $\xi^{N}$ in terms of these basis functions, we obtain

$$
\begin{aligned}
\phi^{N}(t, x, y) & =\sum_{k=1}^{m} \phi_{k}^{N}(t) \psi_{k}^{m}(x, y), & \theta^{N}(t, x, y) & =\sum_{k=1}^{m} \theta_{k}^{N}(t) \psi_{k}^{m}(x, y), \\
u_{j}^{N}(t, x) & =\sum_{i=1}^{n} u_{j, i}^{N}(t) w_{i}^{n}(x), & v_{j}^{N}(t, x) & =\sum_{i=1}^{n} v_{j, i}^{N}(t) w_{i}^{n}(x), j=1,2
\end{aligned}
$$

From equation (1.17), we can write the following first order approximate system

$$
\mathcal{M}^{N} \dot{\bar{\xi}}^{N}(t)=\mathcal{A}^{N} \bar{\xi}^{N}(t)
$$

where $\bar{\xi}^{N}(t)=\left(\bar{\phi}^{N}(t), \bar{\theta}^{N}(t), \bar{u}_{1}^{N}(t), \bar{v}_{1}^{N}(t), \bar{u}_{2}^{N}(t), \bar{v}_{2}^{N}(t)\right) \in R^{N}$, with $N=$ $2(m+2 n)$ is the coordinate vector representation of the approximate state. tion:

We represent the linear system (1.18) more explicitly as the following equa-

$$
\begin{gathered}
{\left[\begin{array}{cccccc}
K_{a}^{N} & 0 & 0 & 0 & 0 & 0 \\
0 & M_{a}^{N} & 0 & 0 & 0 & 0 \\
0 & 0 & K_{b}^{N} & 0 & 0 & 0 \\
0 & 0 & 0 & M_{b}^{N} & 0 & 0 \\
0 & 0 & 0 & 0 & K_{b}^{N} & 0 \\
0 & 0 & 0 & 0 & 0 & M_{b}^{N}
\end{array}\right] \frac{d}{d t}\left[\begin{array}{c}
\bar{\phi}^{N}(t) \\
\bar{\theta}^{N}(t) \\
\bar{u}_{1}^{N}(t) \\
\bar{v}_{1}^{N}(t) \\
\bar{u}_{2}^{N}(t) \\
\bar{v}_{2}^{N}(t)
\end{array}\right]} \\
=\left[\begin{array}{cccccc}
0 & K_{a}^{N} & 0 & 0 & 0 & 0 \\
-K_{a}^{N} & -A_{a}^{N} & 0 & -A_{a b}^{N} & 0 & -A_{a b}^{N} \\
0 & 0 & 0 & K_{b}^{N^{b}} & 0 & 0 \\
0 & A_{a b}^{N} T_{b} & -K_{b}^{N} & -A_{b}^{N} & 0 & 0 \\
0 & 0 & 0 & 0 & 0 & K_{b}^{N} \\
0 & A_{a b}^{N} T & 0 & 0 & -K_{b}^{N} & -A_{b}^{N}
\end{array}\right]\left[\begin{array}{c}
\bar{\phi}^{N}(t) \\
\bar{\theta}^{N}(t) \\
\bar{u}_{1}^{N}(t) \\
\bar{v}_{1}^{N}(t) \\
\bar{u}_{2}^{N}(t) \\
\bar{v}_{2}^{N}(t)
\end{array}\right] .
\end{gathered}
$$

The component matrices can be computed from the sesquilinear forms given by $(1.11)$ :

$$
\begin{array}{cc}
{\left[K_{a}^{N}\right]_{k, \ell}=\sigma_{a}\left(\psi_{k}^{m}, \psi_{\ell}^{m}\right)} & {\left[K_{b}^{N}\right]_{i, j}=\sigma_{b}\left(w_{i}^{n}, w_{j}^{n}\right)} \\
{\left[M_{a}^{N}\right]_{k, \ell}=\int_{\Omega} \psi_{k}^{m} \psi_{\ell}^{m} d x d y \quad\left[M_{b}^{N}\right]_{i, j}=\int_{-1}^{1} w_{i}^{n}(s) w_{j}^{n}(s) d s} \\
{\left[A_{a}^{N}\right]_{k, \ell}=\gamma_{a}\left(\psi_{k}^{m}, \psi_{\ell}^{m}\right) \quad\left[A_{a b}^{N}\right]_{i, \ell}=\rho_{1}\left(w_{i}^{n}, \psi_{\ell}^{m}\right) \quad\left[A_{b}^{N}\right]_{i, j}=\gamma_{b}\left(w_{i}^{n}, w_{j}^{n}\right)}
\end{array}
$$

\section{NUMERICAL RESULTS}

In this section, we demonstrate that the approximate solutions obtained from the Galerkin method described in the previous section have a uniform rate of 
decay. In carrying out this goal, our approach is to calculate the eigenvalues of the matrix

$$
A^{N}=\left(\mathcal{M}^{N}\right)^{-1} \mathcal{A}^{N},
$$

for increasing values of $N$, and observe the trend in the location of the eigenvalues, and the margin of stability which is defined as the magnitude of the maximum of the real part of the eigenvalues. These computations are performed using MATLAB on a SPARC 10 workstation.

For the parameters, we chose $\alpha_{1}=\alpha_{2}=0.1$, and $E$ and $C$ to be 1 . We calculated the eigenvalues and the margin of stability using the eig routine in MATLAB. The results summarized in the table below, clearly indicate that a uniform margin of stability is preserved as the dimension of approximation is increased.

\begin{tabular}{|c|c|}
\hline$N_{a}=N_{b}$ & $\max \left\{\operatorname{Re} \lambda, \lambda \in \sigma\left(A^{N}\right)\right\}$ \\
\hline 10 & -0.07786 \\
14 & -0.05948 \\
18 & -0.05372 \\
22 & -0.05174 \\
26 & -0.05092 \\
\hline
\end{tabular}

\section{References}

[1] Avalos, G. The exponential stability of a coupled hyperbolic/parabolic system arising in structural acoustics. Abstract Appl. Analysis, to appear.

[2] Avalos, G. and Lasiecka, I. The strong stability of a semigroup arising from a coupled hyperbolic/parabolic system. Semigroup Forum, to appear.

[3] Banks, H. T., Fang, W., Silcox, R.J., and Smith, R. C. (1993). Approximation methods for control of structural acoustic models with piezoceramic actuators. Journal of Intelligent Material Systems and Structures, 4:98-116.

[4] Fahroo, F. and Wang, C. (1996) A new model for acoustic-structure interaction and its exponential stability. The Applied Mathematics Quarterly, to appear.

[5] Fahroo, F. and Wang, C. (1998). Convergence and uniform exponential stability of the approximations of acoustic-structure model equations. in preparation.

[6] Micu, S. and Zuazua, E. (1996). Stabilization and periodic solutions of a hybrid system arising in the control of noise. Proceedings of the IFIP TC7/WG-7.2 International Conference, Laredo, España, Lecture Notes in Pure and Applied Mathematics. Marcel Dekker, New York, 174:219-230.

[7] Micu, S. and Zuazua, E. (1998). On a weakly damped system arising in the control of noise. International Series of Numerical Mathematics, Birkhäuser Verlag, Basel. 126:207-222. 